This is an electronic reprint of the original article. This reprint may differ from the original in pagination and typographic detail.

\author{
Author(s): Klintefjord, M.; Hadynska-Klek, K.; Samorajczyk, J.; Görgen, A.; Droste, Ch.; Srebrny, J.; \\ Abraham, T.; Bauer, C.; Garrote, F.L. Bello; Bönig, S.; Damyanova, A.; Giacoppo, F.; \\ Grodner, E.; Hoff, P.; Kisielinski, M.; Komorowska, M.; Korten, W.; Kowalczyk, M.; \\ Kownacki, J.; Larsen, A.C.; Lutter, R.; Marchlewski, T.; Napiorkowski, P.; Pakarinen, \\ Janne; Rapisarda, E.; Reiter, P.; Renstrøm, T.; Siebeck, B.; Siem, S.; Stolarz, A.; \\ Title: $\quad$ Spectroscopy of Low-lying States in $140 \mathrm{Sm}$
}

Year: $\quad 2015$

Version:

Please cite the original version:

Klintefjord, M., Hadynska-Klek, K., Samorajczyk, J., Görgen, A., Droste, Ch., Srebrny, J., Abraham, T., Bauer, C., Garrote, F. B., Bönig, S., Damyanova, A., Giacoppo, F., Grodner, E., Hoff, P., Kisielinski, M., Komorowska, M., Korten, W., Kowalczyk, M., Kownacki, J., . . Zielinska, M. (2015). Spectroscopy of Low-lying States in 140Sm. Acta Physica Polonica B, 46(3), 607-611. https://doi.org/10.5506/APhysPolB.46.607

All material supplied via JYX is protected by copyright and other intellectual property rights, and duplication or sale of all or part of any of the repository collections is not permitted, except that material may be duplicated by you for your research use or educational purposes in electronic or print form. You must obtain permission for any other use. Electronic or print copies may not be offered, whether for sale or otherwise to anyone who is not an authorised user. 


\title{
SPECTROSCOPY OF LOW-LYING STATES IN ${ }^{140} \mathrm{Sm}^{*}$
}

\author{
M. Klintefjord ${ }^{\mathrm{a}}$, K. HADYŃSKa-KLęK ${ }^{\mathrm{a}}$, J. SAmORAJCZYK ${ }^{\mathrm{b}}$
}

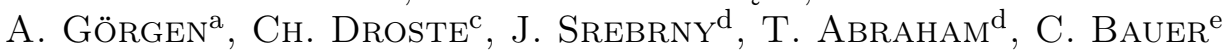
F.L. Bello Garrote ${ }^{a}$, S. Bönig ${ }^{\text {e }}$, A. Damyanova ${ }^{\text {f }}$, F. Giacoppo ${ }^{a}$

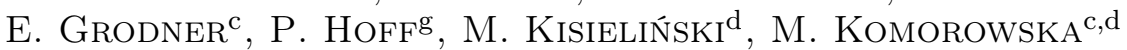
W. Korten ${ }^{h}$, M. KowAlCZYK ${ }^{\text {d }}$, J. KownACKI ${ }^{\text {d,i }}$, A.C. LARSEN ${ }^{\mathrm{a}}$

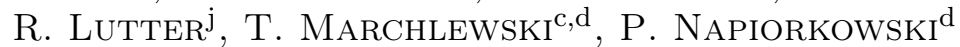
J. PAKARINen $^{\mathrm{k}}$, E. RAPisarda ${ }^{\mathrm{l}, \mathrm{m}}$, P. Reiter ${ }^{\mathrm{n}}$, T. Renstr $\varnothing \mathrm{M}^{\mathrm{a}}$

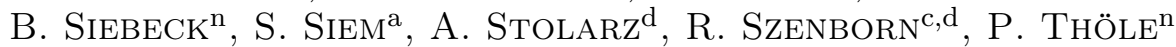

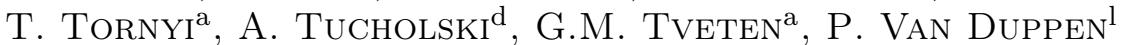
M.J. Vermeulen ${ }^{\mathrm{o}}, \mathrm{N}$. $\mathrm{WARR}^{\mathrm{n}}, \mathrm{H}$. De Witte $^{\mathrm{l}}$, M. Zielińska $^{\mathrm{h}}$

${ }^{a}$ Department of Physics, University of Oslo, 0316 Oslo, Norway

${ }^{\mathrm{b}}$ Faculty of Physics, University of Lodz, 90-236 Łódź, Poland ${ }^{c}$ Faculty of Physics, University of Warsaw, 02-093 Warszawa, Poland ${ }^{\mathrm{d}}$ Heavy Ion Laboratory, University of Warsaw, 02-093 Warszawa, Poland e Institut für Kernphysik, Technische Universität Darmstadt Darmstadt 64289, Germany

${ }^{\mathrm{f}}$ University of Geneva, Bd du Pont-d'Arve 40, 1211 Geneva, Switzerland ${ }^{g}$ Department of Chemistry, University of Oslo, 0316 Oslo, Norway ${ }^{\mathrm{h}}$ CEA Saclay, IRFU, SPHN, 91191 Gif-sur-Yvette, France

${ }^{i}$ National Centre for Nuclear Research, Swierk, 05-400 Otwock, Poland jTechnische Universität München, Garching 85748, Germany

${ }^{k}$ Department of Physics, University of Jyväskylä, Jyväskylä 40014, Finland ${ }^{1}$ Instituut voor Kern- en Stralingsfysica, KU Leuven, Leuven 3001, Belgium ${ }^{\mathrm{m}}$ CERN-ISOLDE, PH Department, CERN, 1211 Geneva 23, Switzerland ${ }^{\text {n} I n s t i t u t ~ f u ̈ r ~ K e r n p h y s i k, ~ U n i v e r s i t a ̈ t ~ z u ~ K o ̈ l n, ~ K o ̈ l n ~ 50937, ~ G e r m a n y ~}$ ${ }^{\circ}$ Department of Physics, University of York, York YO10 5DD, UK

\section{(Received March 23, 2015)}

Electromagnetic transition strengths and spectroscopic quadrupole moments for ${ }^{140} \mathrm{Sm}$ were measured by means of multi-step Coulomb excitation with radioactive beam at the ISOLDE facility at CERN. A complementary experiment was performed at the Heavy Ion Laboratory in Warsaw to assign spins for non-yrast states using the angular correlation technique. Based on the new experimental data previous spin assignments need to be revised.

DOI:10.5506/APhysPolB.46.607

PACS numbers: 23.20.En, 23.20.Js, 25.70.De

* Presented at the Zakopane Conference on Nuclear Physics "Extremes of the Nuclear Landscape", Zakopane, Poland, August 31-September 7, 2014. 


\section{Introduction}

The open-shell nuclei with $Z>50$ and $N<82$ are known to have some of the largest ground-state deformations in the nuclear chart [1]. The shapes of the nuclei in this region are expected to be prolate, except for a small island of nuclei with $Z>62$ and $N \approx 78$, which are predicted to be oblate [2]. Nuclei near ${ }^{140} \mathrm{Sm}$ are, therefore, expected to be located in a transitional region between deformed and spherical shapes (as a function of neutron number) and between prolate and oblate shapes (as a function of proton number), as well as shape coexistence may be expected to occur. Indeed, the rotational bands built on the isomeric $10^{+}$states with $\pi\left(h_{11 / 2}\right)^{2}$ and $\nu\left(h_{11 / 2}\right)^{-2}$ configuration are interpreted as prolate and oblate structures, respectively [3]. Furthermore, an excited state at $990 \mathrm{keV}$ was tentatively assigned as $\left(0^{+}\right)$in ${ }^{140} \mathrm{Sm}[4]$, which could be interpreted as a sign for shape coexistence. The measurement of spectroscopic quadrupole moments and transition strengths for low-lying states represents a sensitive test for theoretical predictions in this region. Electromagnetic transition rates were unknown due to the difficulty of measuring lifetimes below long-lived isomeric states. To determine the transition rates between low-lying states and measure spectroscopic quadrupole moments, a low-energy Coulomb excitation experiment with a radioactive ${ }^{140} \mathrm{Sm}$ beam was performed.

\section{Coulomb excitation experiment}

A quasi-pure ${ }^{140} \mathrm{Sm}$ beam with an average intensity of $2 \times 10^{5}$ particles per second and energy $2.85 \mathrm{MeV}$ per nucleon was produced at ISOLDE using resonant laser ionization and scattered on a ${ }^{94}$ Mo target. The MINIBALL (HPGe) spectrometer [5] coupled to an annular DSSSD array was used for $\gamma$-particle coincidence detection. The DSSSD was placed $25.2 \mathrm{~mm}$ from the target and covered the angular range from $19.7^{\circ}$ to $58.4^{\circ}$ in the laboratory frame. Three excited states in ${ }^{140} \mathrm{Sm}$ were populated during the experiment: the $2^{+}$and $4^{+}$states of the ground-state band and the tentatively assigned $\left(0^{+}\right)$state at $990 \mathrm{keV}$ excitation energy. Figure 1 shows the $\gamma$-ray spectrum taken in coincidence with recoiling ${ }^{94}$ Mo target nuclei detected in the silicon detector. The spectrum was background subtracted and Doppler corrected for the velocity of the scattered ${ }^{140} \mathrm{Sm}$ projectile. The excitation of the $2^{+}$ state at $871 \mathrm{keV}$ in ${ }^{94} \mathrm{Mo}$ appears as a broad structure. The yield from the target excitation is obtained after applying a proper Doppler correction. Since the matrix elements for ${ }^{94} \mathrm{Mo}$ are well known, the yield from the target excitation can be used to normalize the yield from the projectile excitation.

For the analysis, the data was divided into five subsets corresponding to different ranges of scattering angles, and a set of matrix elements was obtained by using a least-square fitting procedure to reproduce the experi- 

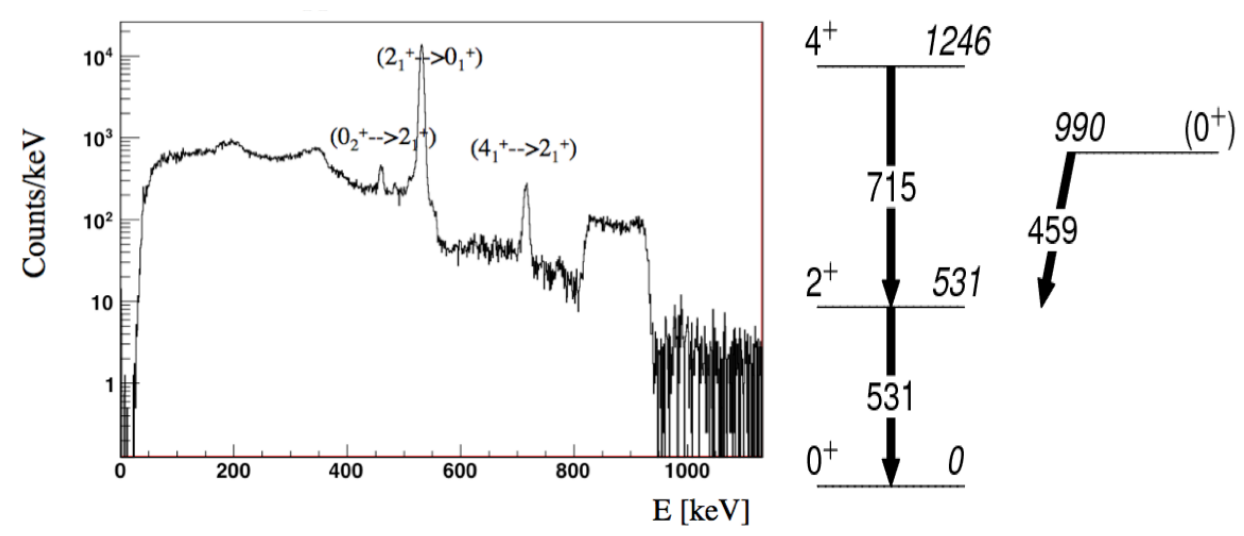

Fig. 1. Background subtracted $\gamma$-ray spectrum, Doppler corrected for the velocity of the scattered ${ }^{140} \mathrm{Sm}$ projectile, and partial level scheme [6].

mentally observed $\gamma$-ray yields with the coupled channels codes GOSIA and GOSIA2 [7]. In this way, the $B\left(\mathrm{E} 2 ; 2_{1}^{+} \rightarrow 0_{1}^{+}\right)$and $B\left(\mathrm{E} 2 ; 4_{1}^{+} \rightarrow 2_{1}^{+}\right)$values could be obtained with high precision. The spectroscopic quadrupole moment of the $2^{+}$state was found to be small and negative. Although the error bars are large, the result suggests a small prolate deformation. The sensitivity to the quadrupole moment was enhanced by using the lifetime of the first excited $2^{+}$state, which was measured recently in a complementary experiment at the Heavy Ion Laboratory in Warsaw with the recoil distance Doppler shift technique using Bucharest-Köln Plunger [8]. The GOSIA analysis resulted in a very large $B\left(\mathrm{E} 2 ; 0_{2}^{+} \rightarrow 2_{1}^{+}\right)$value of more than 200 Weisskopf units, which is difficult to understand. A new experiment was performed at the Heavy Ion Laboratory (Warsaw) to further investigate the nature of the non-yrast states and, in particular, to verify the spin assignments, which in the case of the $990 \mathrm{keV}$ state was based on the non-observation of a direct transition to the ground state [4].

\section{Angular correlation experiment}

Excited states in ${ }^{140} \mathrm{Sm}$ were populated following the ${ }^{140} \mathrm{Eu} \rightarrow{ }^{140} \mathrm{Sm}$ and ${ }^{140} \mathrm{Gd} \rightarrow{ }^{140} \mathrm{Eu} \rightarrow{ }^{140} \mathrm{Sm} \beta$-decays. The ${ }^{140} \mathrm{Eu}$ and ${ }^{140} \mathrm{Gd}$ nuclei were produced in the reactions ${ }^{112} \mathrm{Cd}\left({ }^{32} \mathrm{~S}, p 3 n\right)$ and ${ }^{112} \mathrm{Cd}\left({ }^{32} \mathrm{~S}, 4 n\right)$, respectively, at a beam energy of $155 \mathrm{MeV}$. The recoils were stopped with an Au foil in the center of the EAGLE array [9], which comprised 12 escape-suppressed HPGe detectors. The macrostructure of the beam from the U200P cyclotron with $2 \mathrm{~ms}$ beam on and $4 \mathrm{~ms}$ beam off was used to suppress prompt $\gamma$ rays and only select decay events during the beam-off periods. The detectors of the EAGLE 
array were placed at five relative angles of 42(2), 70(2), 110(2), 138(2) and $180(2)$ degrees. This allowed the analysis of angular correlations for cascades originating from non-yrast states, where a coincidence gate was placed on the $531 \mathrm{keV} 2_{1}^{+} \rightarrow 0_{1}^{+}$transition. Angular correlation coefficients $A_{22}$ and $A_{44}$ were fitted to the observed intensities at different relative angles for the $459-531 \mathrm{keV}$ and the $1068-531 \mathrm{keV}$ cascades. The resulting coefficients are shown in Fig. 2 together with the expected values for $0^{+} \rightarrow 2^{+} \rightarrow 0^{+}$ and $2^{+} \rightarrow 2^{+} \rightarrow 0^{+}$cascades. In the latter case, the angular correlation coefficients depend on the M1/E2 mixing ratio $\delta$ of the $2^{+} \rightarrow 2^{+}$transition.

The results of the angular correlation analysis clearly show the $2^{+}$nature of the state at $990 \mathrm{keV}$, contrary to the previous assignment as a $0^{+}$state. The coefficients are consistent with a mostly pure E2 character of the $459 \mathrm{keV}$ transition. Moreover, the results show that the state at $1599 \mathrm{keV}$ is indeed a $0^{+}$state, contrary to the previous $2^{+}$assignment. The resulting new level scheme is also shown in Fig. 2.
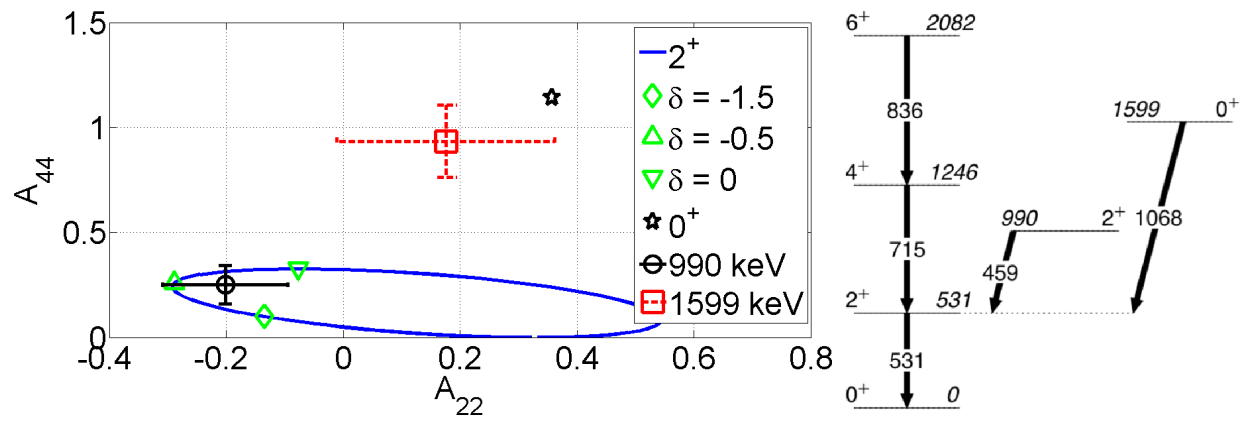

Fig. 2. Angular correlation coefficients for the $\gamma$-cascade from the $990 \mathrm{keV}$ (black circle) and from the $1599 \mathrm{keV}$ state (red square). The closed curve shows expected values for a $2^{+} \rightarrow 2^{+} \rightarrow 0^{+}$cascade for different M1/E2 mixing ratios $\delta$. The symbols (gray/green) on the black curve indicate the angular correlation coefficients for specific values of $\delta$. The star indicates the expected coefficients for a $0^{+} \rightarrow 2^{+} \rightarrow$ $0^{+}$cascade.

\section{Conclusions}

The information obtained from the angular correlation measurement necessitates a re-analysis of the Coulomb excitation data with the correct spin assignments. The finding that the state at $990 \mathrm{keV}$ has spin-parity $2^{+}$requires that the $\left\langle 2_{2}^{+}\|\mathrm{M} 1\| 2_{1}^{+}\right\rangle,\left\langle 2_{2}^{+} \| \mathrm{E} 2|| 2_{2}^{+}\right\rangle$and $\left\langle 2_{2}^{+}\|\mathrm{E} 2\| 0_{1}^{+}\right\rangle$matrix elements are included in the analysis. Preliminary results of the new GOSIA analysis suggest that the $B\left(\mathrm{E} 2 ; 4_{1}^{+} \rightarrow 2_{1}^{+}\right)$and $B\left(\mathrm{E} 2 ; 2_{1}^{+} \rightarrow 0_{1}^{+}\right)$values are insensitive to the new spin assignment. The $B\left(\mathrm{E} 2 ; 2_{2}^{+} \rightarrow 2_{1}^{+}\right)$value corresponding to 
the $459 \mathrm{keV}$ transition, on the other hand, changes significantly toward a smaller value. The $B\left(\mathrm{E} 2 ; 2_{2}^{+} \rightarrow 0_{1}^{+}\right)$value is found to be very small, which is in agreement with the non-observation of the transition in the present and previous experiments. Including the M1/E2 mixing ratio from the angular correlation experiment as known spectroscopic data in the Coulomb excitation analysis does not yield more precise matrix elements due to its relatively large uncertainty. The analysis of the Coulomb excitation data is presently being finalized and results will be presented and compared to theoretical calculations in a forthcoming publication.

This work was supported in part by the Research Council of Norway, project 213442, BMBF, Germany, under contract 05P12PKFNE, and by the European Union Seventh Framework Programme through ENSAR, contract No. RII3-CT-2010-262010.

\section{REFERENCES}

[1] P. Moeller et al., At. Data. Nucl. Data Tables 59, 185 (1995).

[2] J.-P. Delaroche et al., Phys. Rev. C81, 014303 (2010).

[3] M.A. Cardona et al., Phys. Rev. C44, 891 (1991).

[4] R.B. Firestone et al., Phys. Rev. C43, 1066 (1991).

[5] N. Warr et al., Eur. Phys. J. A49, 40 (2013).

[6] N. Nica, Nucl. Data Sheets 108, 1287 (2007).

[7] T. Czosnyka, D. Cline, C.Y. Wu, Bull. Am. Phys. Soc. 28, 745 (1983).

[8] F.L. Bello Garrote et al., to be published.

[9] J. Mierzejewski et al., Nucl. Instrum. Methods A659, 84 (2011). 